\title{
Repeat Administration of an Adenovirus Vector Encoding Cystic Fibrosis Transmembrane Conductance Regulator to the Nasal Epithelium of Patients with Cystic Fibrosis
}

\author{
Joseph Zabner, ${ }^{\star}$ Bonnie W. Ramsey, ${ }^{\ddagger}$ David P. Meeker, ${ }^{\S}$ Moira L. Aitken, ${ }^{\ddagger}$ Rosemary P. Balfour, ${ }^{\text {ๆ }}$ Ronald L. Gibson, ${ }^{\ddagger}$ \\ Jan Launspach, ${ }^{*}$ Richard A. Moscicki, ${ }^{\S}$ Susan M. Richards, ${ }^{\S}$ Thomas A. Standaert, ${ }^{\ddagger}$ Judy Williams-Warren, ${ }^{\ddagger}$ \\ Samuel C. Wadsworth, ${ }^{\S}$ Alan E. Smith, ${ }^{\S}$ and Michael J. Welsh* \\ *Howard Hughes Medical Institute, Departments of Internal Medicine and Physiology and Biophysics, University of Iowa College of \\ Medicine, Iowa City, Iowa 52242; ${ }^{\circ}$ Departments of Pediatrics and Medicine, University of Washington, and University of Washington \\ Medical Center, Seattle, Washington 98105; and ${ }^{\S}$ Genzyme Corporation, Framingham, Massachusetts 01701
}

\begin{abstract}
Cystic fibrosis (CF) is a common autosomal recessive disease caused by mutations in the CF transmembrane conductance regulator gene. Recombinant adenoviruses have shown promise as vectors for transfer of CF transmembrane conductance regulator cDNA to airway epithelia and correction of the $\mathrm{Cl}^{-}$transport defect. However, because adenovirus-mediated gene transfer is transient, use of adenovirus as a vector for treatment of $\mathrm{CF}$ would require repeated administration. Therefore, we evaluated repeat administration of an adenovirus vector to the nasal epithelium of patients with $\mathrm{CF}$ with five escalating doses of up to $10^{10}$ infectious units. There were no detectable adverse affects. All subjects were initially seropositive but developed additional humoral immune responses. The vector partially corrected the defect in airway epithelial $\mathrm{Cl}^{-}$transport in some subjects, although there was variability between subjects and there was less correction with subsequent administration, perhaps because the immune response limited gene transfer. Future work must focus on vectors with increased efficiency and with the ability to evade host defenses. (J. Clin. Invest. 1996. 97:1504-1511.) Key words: gene transfer • airway • immune response $\bullet$ chloride $\bullet$ ion channel
\end{abstract}

\section{Introduction}

Cystic fibrosis $(\mathrm{CF})^{1}$ is a common autosomal recessive disease caused by mutations in the gene for the $\mathrm{CF}$ transmembrane

Address correspondence to Michael J. Welsh, M.D., Howard Hughes Medical Institute, University of Iowa College of Medicine, 500 EMRB, Iowa City, IA 52242. Phone: 319-335-7619; FAX: 319-3357623.

Received for publication 1 August 1995 and accepted in revised form 3 January 1996.

1. Abbreviations used in this paper: $\mathrm{CF}$, cystic fibrosis; $\Delta V_{\mathrm{t}}$, change in voltage across the nasal epithelium; $\Delta V_{\mathrm{t}}$ Amiloride, change in $V_{\mathrm{t}}$ produced by perfusing the mucosal surface of the epithelium with amiloride; $\Delta V_{\mathrm{t} \text { Low Cl}}$, change in $V_{\mathrm{t}}$ produced by perfusion of the mucosal surface with a solution containing terbutaline and a low $\mathrm{Cl}^{-}$ concentration in the continued presence of amiloride; I.U., infectious units.

J. Clin. Invest.

(C) The American Society for Clinical Investigation, Inc.

0021-9738/96/03/1504/08 \$2.00

Volume 97, Number 6, March 1996, 1504-1511 conductance regulator (CFTR) (1). CFTR is a $\mathrm{Cl}^{-}$channel that is regulated by phosphorylation and that mediates $\mathrm{Cl}^{-}$ transport across epithelia in the lung, intestine, pancreas, and sweat gland (2-4). The loss of CFTR function in CF epithelia leads to lesions in all of these organs, but disease of the pulmonary airways is now the main cause of morbidity and mortality (1). Although current treatments have improved the lives of patients, no treatment is directed at the underlying defect, and $\mathrm{CF}$ remains a lethal disease. For this reason, there have been increasing efforts directed at developing gene transfer to airway epithelia as a potential treatment.

In vitro studies have shown that recombinant adenovirus vectors can express CFTR in cultured airway epithelia and can correct the $\mathrm{Cl}^{-}$transport defect and associated increase in $\mathrm{Na}^{+}$ transport that characterize CF airway epithelia (5-8). Since the first in vivo studies reporting that an adenovirus vector can express CFTR in the airway epithelia of cotton rats (5), a number of studies in animals and in tracheal explants have shown that adenovirus vectors can express CFTR as well as reporter genes in airway epithelia in vivo (9-11). Although there is little doubt that adenovirus vectors can deliver genes, both in vitro and in vivo, the efficacy and specificity of this process is uncertain (12). Some gene transfer studies suggest the need for very high MOI's and suggest that some cells are more susceptible to adenovirus vector-mediated gene transfer than others.

Adenovirus vectors have been delivered to patients with CF where they express CFTR protein and partially correct the $\mathrm{CF}$ defect in electrolyte transport $(13,14)$. However, because adenovirus-mediated gene transfer is transient, use of recombinant adenovirus as a vector for treatment of $\mathrm{CF}$ would require repeated administration. This requirement is of concern because, in both animals and humans, administration of adenovirus can be associated with an inflammatory response that may limit gene expression (14-22). Furthermore, repeated administration has resulted in the development of neutralizing antibodies, at least in animals (21).

The present work was designed as a phase I study to test the safety of repeat administration of an adenovirus vector to airway epithelium of patients with CF. We chose the nasal epithelium for three reasons. First, access is simple and noninvasive, an important consideration in a trial involving repeated instrumentation and measurements over an extended period of time. Second, the safety of repeated adenovirus administration to humans is unknown, and we wished to minimize the risk to participants if an adverse event should occur. Third, nasal airway epithelia have a similar histology and the same CFassociated abnormalities in electrolyte transport as pulmonary epithelia. We measured changes in voltage across the nasal ep- 
ithelium $\left(\Delta V_{\mathrm{t}}\right)$ as an indication of the ability of the vector to correct CF electrolyte transport abnormalities $(23,24)$.

\section{Methods}

\section{Subjects}

We studied six patients who ranged from 24 to $54 \mathrm{yr}$ of age (Table I). The diagnosis of $\mathrm{CF}$ was based on measurement of the sweat $\mathrm{Cl}^{-}$concentration, evaluation of lung and pancreatic disease, and genotyping of the CFTR gene.

\section{Study design}

The study was approved by the University of Iowa and the University of Washington Institutional Review Boards and Institutional Biosafety Committees. It was also approved by the National Institutes of Health Recombinant DNA Advisory Committee and the Food and Drug Administration.

The design of the study and the timing of evaluations are described in detail in reference 25 . Each subject received multiple administrations of Ad2/CFTR-2, with each successive dose higher than the last. The doses were $2 \times 10^{7}$ infectious units (I.U.), $2 \times 10^{8}$ I.U., $2 \times 10^{9}$ I.U., $6 \times 10^{9}$ I.U., and $1 \times 10^{10}$ I.U. The minimum duration between doses was $28 \mathrm{~d}$. The average interval between doses was $44 \pm 10($ mean \pm SD) d. The first four subjects received all five administrations of Ad2/CFTR-2. Because there were no adverse events associated with the lowest dose and because there was little evidence for electrophysiologic changes at that dose, the last two subjects received $2 \times 10^{8}$ I.U. as their first dose and received a total of four doses. All six subjects completed the study.

At the start of the study, one nostril was chosen at random to be the "treated" nostril, which received Ad2/CFTR-2. The other nostril was chosen as the "mock" nostril, which was treated and evaluated identically with the exception that the PBS vehicle was administered instead of Ad2/CFTR-2. During the study of the first two subjects (A01 and A02), the investigators and the subjects were aware of which nostril was treated and which was mock. For study of the last four subjects (B01-B04), both investigators and subjects were blinded as to which nostril was treated and which was mock. Data from all six subjects were evaluated in a blinded fashion.

\section{Adenovirus vector}

The vector used was Ad2/CFTR-2, an adenovirus 2-based E1 replacement vector containing the CFTR cDNA, the PGK promoter, and a synthetic bovine growth hormone poly $\mathrm{A}$ addition site. The vector retains the $\mathrm{E} 3$ region but lacks all of $\mathrm{E} 4$ with the exception of ORF6 (25). Vector was grown in 293 cells, purified using $\mathrm{CsCl}$ gradient centrifugation, and shown to contain $<1$ replication competent

\section{Table I. Characteristics of Participants}

\begin{tabular}{|c|c|c|c|c|c|c|}
\hline Subject & Sex & Age & CF mutation & Sweat [C1] & $\begin{array}{c}\text { FEV1 } \\
\text { (\% predicted) }\end{array}$ & $\begin{array}{l}\text { Pancreatic } \\
\text { function }\end{array}$ \\
\hline & & $y r$ & & $m M$ & & \\
\hline A01 & $\mathrm{M}$ & 31 & $\Delta F 508 / 3849+10$ & 26 & 67 & PS \\
\hline A02 & M & 42 & $\Delta \mathrm{F} 508 / ?$ & 104 & 54 & PI \\
\hline B01 & M & 31 & $\Delta \mathrm{F} 508 / \Delta \mathrm{F} 508$ & 121 & 83 & PI \\
\hline B02 & M & 28 & $\Delta \mathrm{F} 508 / \Delta \mathrm{F} 508$ & 114 & 96 & PI \\
\hline B03 & M & 54 & $\Delta \mathrm{F} 508 / ?$ & 81 & 83 & PI \\
\hline B04 & $\mathrm{F}$ & 24 & $\Delta \mathrm{F} 508 / \mathrm{R} 506 \mathrm{~T}$ & 118 & 91 & PI \\
\hline
\end{tabular}

For CF mutation,? refers to an unknown mutation. PS and PI refer to pancreatic sufficient and pancreatic insufficient, respectively. Subjects A01 and A02 were enrolled at the University of Iowa and subjects B01B04 at the University of Washington. adenovirus (RCA) per patient dose of vector, except at the highest dose, when $<10$ RCA were present per dose.

\section{Vector administration}

Vector was administered to subjects while they were supine. No anesthetics or vasoconstrictors were used. We applied vector to the nasal epithelium using a modified Foley catheter, which was introduced under endoscopic guidance to the area beneath the inferior turbinate. The catheter was positioned at the same depth beneath the inferior turbinate, $6 \mathrm{~cm}$ from the most caudal aspect of the columella, for each dose and for all $V_{\mathrm{t}}$ measurements. Vector was infused slowly over the course of $30 \mathrm{~min}$. We made no attempt to constrain the vector solution to a small area, and in fact our studies with methylene blue suggest that administered fluid rapidly spreads to cover the entire inferior turbinate and most of the nasal mucosa on the side of administration. Thus, the area to which vector is applied is undefined but is probably at least $10-30 \mathrm{~cm}^{2}$. As a control, saline was administered simultaneously to the contralateral nostril using the same procedure.

The initial titer of Ad2/CFTR-2 was either $1.8 \times 10^{10} \mathrm{I} . \mathrm{U} . / \mathrm{ml}$ or $8.4 \times 10^{10} \mathrm{I}$.U. $/ \mathrm{ml}$. Vector was diluted with PBS to achieve the final dose. Doses of $2 \times 10^{7}$ I.U. to $2 \times 10^{9}$ I.U. and four of the doses of $6 \times 10^{9}$ were administered in a volume of $100 \mu l$. Two of the doses of $6 \times 10^{9}$ I.U. were administered in $200 \mu$ l. Doses of $10^{10}$ I.U. were administered in a volume of 200-600 $\mu \mathrm{l}$.

\section{Measurement of nasal $V_{t}$}

The transepithelial electric potential difference across the nasal epithelium $V_{\mathrm{t}}$ was measured using a modification of previously described methods $(13,23,24)$. The reference electrode was a 25 -gauge $1 / 2$-inch subcutaneous needle connected with sterile normal saline solution to a silver/silver chloride pellet (E.W. Wright, Guilford, CT). The exploring electrode was a pediatric size 8 teflon-coated latex Foley catheter (modified; Rüsch, Inc., Duluth, GA) with two side holes at the tip. The catheter was filled with Ringer's solution containing (in $\mathrm{mM}$ ) $135 \mathrm{NaCl}, 2.4 \mathrm{KH}_{2} \mathrm{PO}_{2}, 0.6 \mathrm{~K}_{2} \mathrm{HPO}_{4}, 1.2 \mathrm{CaCl}_{2}, 1.2 \mathrm{MgCl}_{2}$, and 10 Hepes (titrated to $\mathrm{pH} 7.4$ with $\mathrm{NaOH}$ ) and was connected to a silver/ silver chloride pellet. Voltage was measured with a voltmeter (Keithley Instruments Inc., Cleveland, $\mathrm{OH}$ ) connected to a strip chart recorder (Servocorder; Western Graphtec Inc., Irvine, CA). Before making measurements, the silver/silver chloride pellets were connected in series with the Ringer's solution, and zero voltage was established; the pellets were changed if the offset voltage was $> \pm 4$ $\mathrm{mV}$. The rubber catheter was introduced into the nostril under telescopic guidance (Hopkins Telescope; Karl Storz, Tuttlingen, Germany), and the side holes of the catheter were placed under the inferior nasal turbinate $6 \mathrm{~cm}$ from the most caudal aspect of the columella. The catheter was then left in that position for the entire recording period. A continuous flow of solution was used by connecting a 3 to 1 administration set (Trifuse ${ }^{\circledR}$; Medinfusion, Inc., Duluth, GA) to the balloon port of the Foley catheter. Each solution was given by controlled infusion using an infusion pump (model 975; Harvard Apparatus, S. Natick, MA) at a constant rate of $5 \mathrm{ml} / \mathrm{min}$. All perfusing solutions were at room temperature. The following solutions were administered in this order: (a) Ringer's solution; $(b)$ Ringer's solution containing $100 \mu \mathrm{M}$ amiloride (Merck and Co., Inc., West Point, PA); and $(c)$ a low $\mathrm{Cl}^{-}$solution (in which $135 \mathrm{mM} \mathrm{Na}$ gluconate replaced $135 \mathrm{mM} \mathrm{NaCl}$ ) with $100 \mu \mathrm{M}$ amiloride plus $10 \mu \mathrm{M}$ terbutaline (Geigy Pharmaceuticals, Ardsley, NY). Each solution was perfused for a period of at least $5 \mathrm{~min}$. Once a stable $V_{\mathrm{t}}$ reading was obtained, perfusion with the next solution was begun. The entire procedure took $\sim 30-45 \mathrm{~min}$. Measurement of $V_{\mathrm{t}}$ at Iowa City and Seattle used identical equipment and procedures, and records from each site were read blinded at the opposite site. Moreover, investigators from the two sites verified the methods of $V_{\mathrm{t}}$ measurement by traveling to the other site. $7.3 \%$ of $V_{\mathrm{t}}$ measurements could not be interpreted for reasons such as unstable baselines or loss of position of the recording catheter. 


\section{Immunologic assessments}

Adenovirus-specific IgG and IgA by ELISA. ELISAs were developed for the detection of $\operatorname{IgG}$ and $\operatorname{IgA}$ antibodies specific for $\mathrm{Ad} 2 /$ CFTR-2 in patient sera, nasal washes, and control sera. IgG titers were expressed as the reciprocal of the dilution giving an absorbance value $\leq 0.1$. IgA values represent semi-quantitative levels determined by interpolation from an $\operatorname{IgA}$ standard curve.

Adenovirus-neutralizing antibody. Serially diluted, heat-inactivated patient sera, nasal washes, or control sera were preincubated with serotype 2 adenovirus in 96-well tissue culture plates for $1 \mathrm{~h}$ at $37^{\circ} \mathrm{C}$. Subsequently, $100 \mu \mathrm{l}$ of 293 cells $\left(3 \times 10^{5}\right.$ cells $\left./ \mathrm{ml}\right)$ were added, and the cultures were incubated for $3 \mathrm{~d}$ at $37^{\circ} \mathrm{C}$. The wells were examined for inhibition of cytopathic effects. Neutralization titer was defined as the reciprocal of the maximum patient serum dilution that inhibited viral infectivity such that $>90 \%$ of the cells remained viable relative to the controls.

Evaluation of antibodies by Western blot. The profile of patient antibody responses to $\mathrm{Ad} 2$ proteins was evaluated by Western blot. Briefly, Ad2/CFTR-2 viral proteins were separated by SDS-PAGE on a $4-20 \%$ gradient gel, electroblotted onto an Immubilon-P (Millipore, Bedford, MA) transfer membrane, blocked with casein, and probed with patient sera diluted 1/1,000 or control sera. Resultant immune complexes were detected with horseradish peroxidase conjugates specific for human IgG or IgA followed by their visualization with a chemiluminescent substrate. The banding patterns were compared with a gold-stained Western blot of viral proteins.

Human CFTR specific antibodies. An ELISA was developed as a screening assay for the detection of antibodies to CFTR using insectderived recombinant CFTR. Patient sera were tested at a dilution of 1/100. Pooled normal human serum was used as a negative control, and biotinylated rabbit anti-CFTR was the positive control. Biotinylated goat anti-human IgG, IgA, IgM, and streptavidin-horseradish peroxidase were used to detect bound antibody.

\section{Clinical assessments}

History, physical examination, and measurement of serum chemistries and blood counts (25) were performed at the times indicated in reference 25. Pulmonary function tests were performed in accordance with ATS guidelines. Sputum cultures were performed to identify bacterial pathogens using standard microbiological techniques.

\section{Culture for adenovirus}

Cultures to detect Ad2/CFTR-2 were performed on the permissive 293 cell line. Supernatants from swabs were added to 293 cells grown in 96-well plates at $50 \%$ confluence. The 293 cells were incubated for $48 \mathrm{~h}$ at $37^{\circ} \mathrm{C}$ and then fixed and incubated with an FITC-labeled antiadenovirus mAb (Chemicon; Light Diagnostics, Temecuca, CA) for $30 \mathrm{~min}$. Positive nuclear immunofluorescence was interpreted as a positive culture. The sensitivity of the assay was evaluated by dipping the swab in dilutions of Ad2/CFTR-2 suspension, followed by subsequent handling as for patient specimens, including storage, transport, and culture procedure. Positive cultures were detected when the initial viral suspension contained $10^{3}$ I.U./ml.

\section{Measurements of cytology and cytokines in nasal wash}

IL- $1 \beta$ and TNF- $\alpha$ were analyzed using reagents from Genzyme Corp. (Framingham, MA), IL-6 was measured using high-sensitivity reagents (Quantikine; R\&D Systems, Minneapolis, MN), and IL-8 levels were determined with PerSeptive Diagnostics (Cambridge, MA) reagents. Detectable levels of the cytokines were as follows: IL-1 $\beta$, $8 \mathrm{pg} / \mathrm{ml}$; IL-6, $0.63 \mathrm{pg} / \mathrm{ml}$; IL-8, $50 \mathrm{pg} / \mathrm{ml}$; and TNF- $\alpha, 9.4 \mathrm{pg} / \mathrm{ml}$. For cytology, cells obtained by nasal swab were dislodged in $1.5 \mathrm{ml}$ of 293 media without serum. Cells were pelleted at $1,000 \mathrm{rpm}$ for $5 \mathrm{~min}$ and resuspended in $0.5 \mathrm{ml}$ of 293 media. $60 \mu \mathrm{l}$ of the cell suspension was cytocentrifuged onto slides and stained with Wright's stain. Samples were examined by light microscopy, 100-300 cells were counted, and the cell differential determined.

\section{Results and Discussion}

Evaluation for adverse events. We observed no adverse events that could be attributed to delivery of the vector. There were 16 episodes of exacerbation of pulmonary disease in which antibiotics used to treat the CF pulmonary disease were begun or changed during the course of the study. We could discern no temporal relationship between these episodes and vector administration. We believe these events and their frequency are consistent with the predicted course of the disease in these patients over a study period lasting 111-245 d. Measurements of blood cell counts and general chemistries showed no significant alterations in any subject during the course of treatment.

Evaluation for adverse events associated with vector administration to the nasal epithelium is complicated by the fact that patients with $\mathrm{CF}$ have chronic nasal inflammation (26). During the course of study the severity of inflammation varied, but we could discern no relation to vector administration. Neither the subjects nor the investigators were able to identify the treated side by the presence of symptoms or appearance of the mucosa. We also assessed cells obtained by nasal swab. The specimens typically showed a predominance of squamous and respiratory epithelial cells, $0-10 \%$ neutrophils, and 0-5\% lymphocytes. There were no significant changes during the study that could be attributed to vector administration. Measurement of IL- $1 \beta$, IL- 6 , IL- 8 , and TNF- $\alpha$ in the nasal washes were consistently below the sensitivity limits of the assays (0.6-50 $\mathrm{pg} / \mathrm{ml})$.

These data suggest that there were no significant systemic or local adverse events that could be attributed to administration of up to $10^{10}$ I.U. of Ad2/CFTR-2 to the nasal mucosa. The absence of local adverse events is similar to other reports (14). Crystal and colleagues have reported systemic adverse events after lung administration of recombinant adenovirus $(14,15)$. Studies in animals also suggest that adenovirus administered to the lung can cause an inflammatory response, but the effects are dose dependent and vary with different species $(16-19,21)$. Additional studies will be required to evaluate adverse effects in the human lung.

Evaluation for antibody production. All subjects were required to have preexisting antibodies to adenovirus at the time of enrollment. Fig. 1 lists baseline and time-dependent changes in adenovirus-specific IgG serum antibodies and neutralizing antibody titers for each patient. Because absolute antibody titers varied from subject to subject, data are presented as titers normalized to a value of 1 at the start of the study. At doses of $2 \times 10^{9}$ I.U. and higher, there were transient fourfold increases in serum IgG antibody in three of the six subjects (B01, B02, and B03). Three subjects (B02, B03, and B04) had a fourfold increase in serum-neutralizing antibodies. Although these increases are modest, they do suggest that the subjects were mounting an immune response to the vector. That a more substantial change in serum antibody titers was not observed may reflect the preexisting antibody in the patients.

Samples from nasal wash were analyzed for adenovirusspecific IgA antibodies. We were unable to detect a significant change from baseline in antibody titer in samples obtained by nasal washing; however, our inability to do so may have been a result of dilution of a small amount of airway surface fluid by the relatively large volume $(5 \mathrm{ml})$ used for the collection. We have detected an increase in $\operatorname{IgA}$ in nasal washes from mon- 

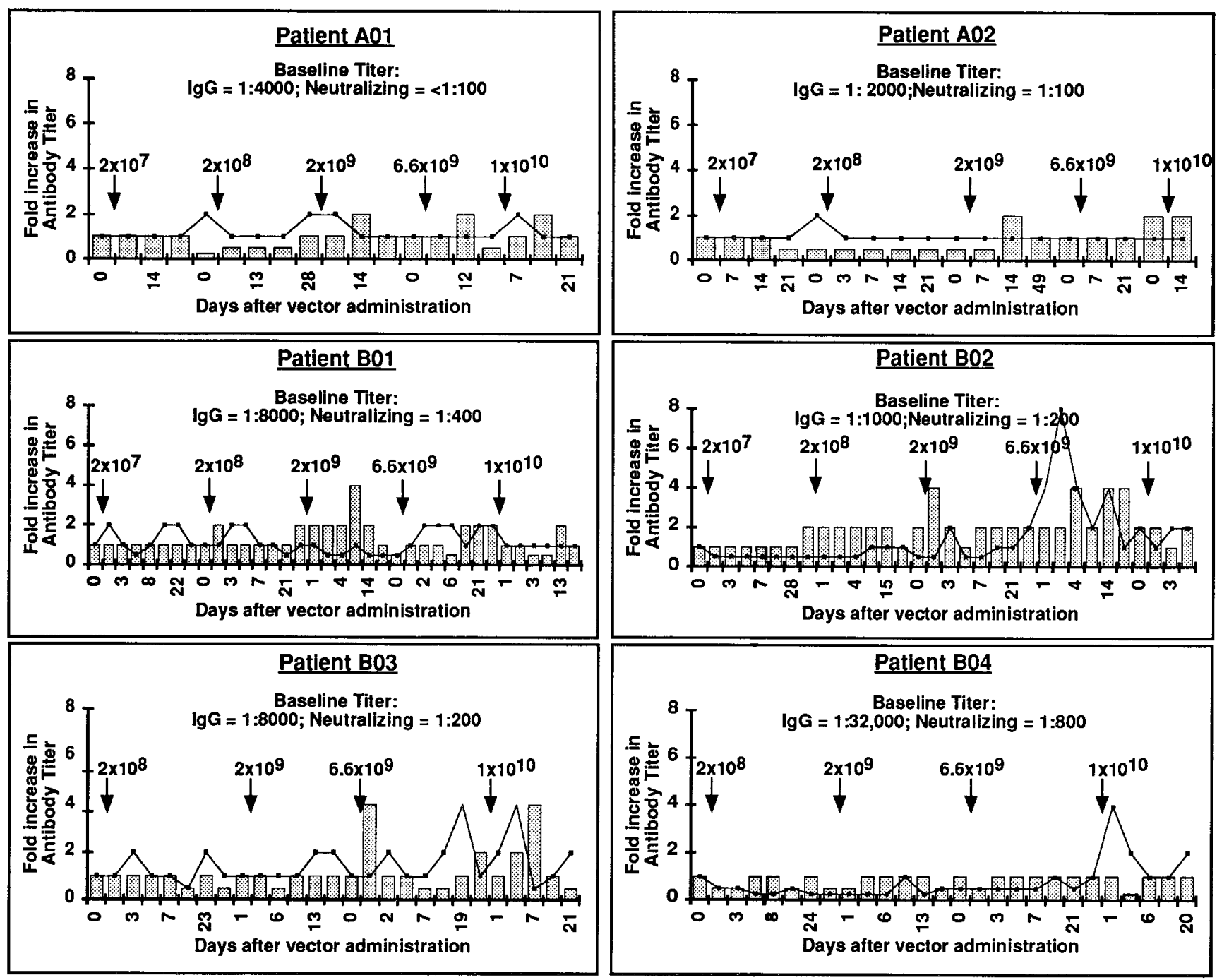

Figure 1. Antibody titers. Each panel shows data from an individual subject. Baseline titers of IgG and neutralizing antibodies are indicated for each subject. Bars show serum IgG titers and filled squares and lines show titers of neutralizing antibody. Data are presented as fold increase in antibody titer with titer at the start of the study equal to 1 . Times and doses of vector administration are indicated by arrows. For uniformity, not all data points are shown.

keys that received intrapulmonary adenovirus (21). In those studies, higher doses of vector (on a per-kilogram basis) were used, and the animals were seronegative before administration.

Although not all of the subjects showed a fourfold increase in serum antibody titers, evaluation of serum by Western blot to adenoviral proteins provided additional evidence that all subjects developed an immunologic response. Fig. 2 shows an example of a Western blot from subject B04, who had no change in serum IgG titers by ELISA. The figure shows a time-dependent quantitative increase in intensity of antibody reaction with several viral proteins. In addition, qualitative changes indicated by recognition of new epitopes were also observed; for example, note the reaction with the core major protein at $\sim 22 \mathrm{kD}$. The pattern of reactivity on Western blot varied from subject to subject; however, there was a quantitative change in $\mathrm{IgG}$ and $\mathrm{IgA}$ reactivity with all subjects except B03, and a qualitative change with subjects B02 and B04. None of the subjects developed antibodies to CFTR (not shown).

Thus, changes in one or the other of the ELISA titers, titer of neutralizing antibodies, and the pattern of reactivity de- tected by Western blot indicate that all subjects developed an additional immune response to the adenoviral proteins as a consequence of vector administration. The response was complex and varied from patient to patient. However, the pattern of immune response did not differentiate patients with either large or absent electrophysiologic changes as described below.

Culture for Ad2/CFTR-2. We cultured material from nasal swabs, sputum, rectal swabs, and urine for Ad2/CFTR-2 on the permissive 293 cell line. Subject B01 had a positive culture from a nasal swab taken $24 \mathrm{~h}$ after administration of $2 \times 10^{9}$ I.U. All other cultures at $24 \mathrm{~h}$ and later time points were negative from all sites. These data suggest that the recombinant vector was rapidly cleared. They also suggest that there was no significant replication of Ad2/CFTR-2. These results are consistent with our earlier studies showing that replication of $\mathrm{Ad} 2 /$ CFTR-2 (27) and of a related vector Ad2/CFTR-1 (6) is severely impaired in tissue culture and in experimental animals.

Measurement of nasal $V_{t}$ in $C F$ and non-CF subjects. The loss of CFTR $\mathrm{Cl}^{-}$channel function causes defective cAMPstimulated $\mathrm{Cl}^{-}$transport across airway epithelia (1). In addi- 


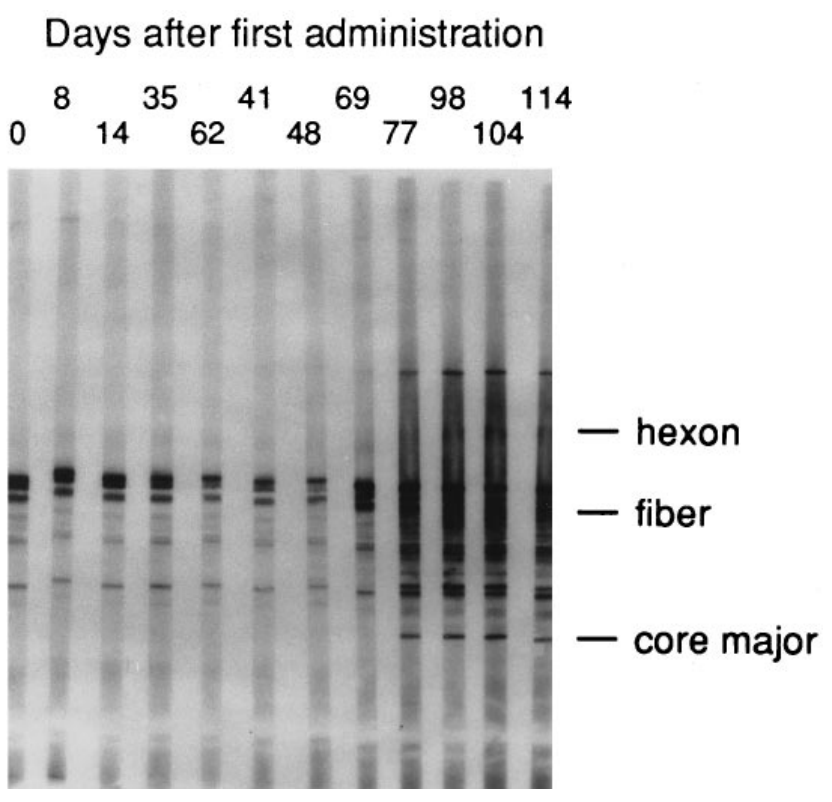

Figure 2. Western blot of serum from subject B04 to adenoviral proteins. Data are an example of the Western blot to detect IgA against adenoviral proteins. Similar results were obtained when IgG was detected.

tion, CF epithelia manifest increased amiloride-sensitive absorption of $\mathrm{Na}^{+}$, although the responsible mechanisms are not fully understood. Because both processes are electrically conductive, they can be assayed qualitatively by measuring $V_{\mathrm{t}}$ in vivo $(23,24)$. In this study, we made three types of measurements. First, we measured basal $V_{\mathrm{t}}$, which is increased in $\mathrm{CF}$ and reflects the increased $\mathrm{Na}^{+}$and absent $\mathrm{Cl}^{-}$transport. Second, we measured the change in $V_{\mathrm{t}}$ produced by perfusing the mucosal surface of the epithelium with amiloride ( $\left.\Delta V_{\mathrm{t} \text { Amiloride }}\right)$. $\Delta V_{\mathrm{t} \text { Amiloride }}$ is increased in $\mathrm{CF}$ and reflects predominately the increased basal $\mathrm{Na}^{+}$transport. Third, we measured the change in $V_{\mathrm{t}}$ produced by perfusion of the mucosal surface with a solution containing terbutaline and a low $\mathrm{Cl}^{-}$concentration in the continued presence of amiloride $\left(\Delta V_{\mathrm{t} \text { Low Cl}}\right) \cdot \Delta V_{\mathrm{t} \text { Low Cl }}$ primarily reflects a $\mathrm{Cl}^{-}$diffusion voltage through $\mathrm{CFTR}^{-}$channels (see also Methods).

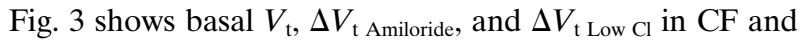
non-CF individuals. As previously reported $(23,24)$, all three measurements distinguish CF from non-CF epithelia, although there is variability in individual measurements.

Evaluation of $V_{t}$ before and after administration of Ad2/ CFTR-2. Because only two of the subjects who received the lowest dose $\left(2 \times 10^{7}\right.$ I.U. $)$ had $V_{\mathrm{t}}$ measured as described above, we focused on measurements made at Ad2/CFTR-2 doses of $2 \times 10^{8}$ I.U. and greater. For each administration, we calculated average values before vector administration and for $1 \mathrm{wk}$ after administration. When measurements made before and after administration were compared, there was substantial variability on both the treated and the mock sides. We observed no consistent or statistically significant changes in basal $V_{\mathrm{t}}$ or $\Delta V_{\mathrm{t} \text { Amiloride }}$ at any of the doses (data not shown).

Fig. 4 shows measurements of $\Delta V_{\mathrm{t} \text { Low Cl }}$ for each individual subject (symbols and lines) and average values $( \pm \mathrm{SEM})$ (bars) before and after administration of each dose of Ad2/CFTR-2. We observed substantial variability between individual sub- jects and different administrations. However, examination of values from individual subjects shows that, in some of the treated epithelia, $V_{\mathrm{t}}$ hyperpolarized after vector administration. The effect of vector administration can be appreciated by noting that, in the mock epithelia (Fig. 4, top), $\Delta V_{\mathrm{t} \text { Low Cl }}$ was rarely negative after administration of vehicle. In contrast, for epithelia that received Ad2/CFTR-2 (Fig. 4, bottom), $\Delta V_{\mathrm{t} \text { Low Cl }}$ after treatment was $<0$ on several occasions.

Examination of values for individual subjects suggests that Ad2/CFTR-2-dependent changes may have been more prominent at the mid doses. These trends are borne out by the average values of $\Delta V_{\mathrm{t} \text { Low Cl }}$ (Fig. 4, bars). At doses of $2 \times 10^{9}$ and $6 \times 10^{9} \mathrm{Ad} 2 / \mathrm{CFTR}-2, \Delta V_{\mathrm{t} \text { Low Cl }}$ after vector delivery was significantly lower than before treatment. At the fifth dose of $1 \times 10^{10}$, the changes in $\Delta V_{\mathrm{t} \text { Low Cl }}$ were less prominent and not statistically significant. Thus, it appeared that there was a trend for Ad2/CFTR-2 to correct $\Delta V_{\mathrm{t} \text { Low } \mathrm{Cl}}$ partially at the mid doses but to have less effect at the lowest dose and at the highest dose.

We speculate that there may have been less correction at the highest dose because development of humoral immunity with neutralizing antibodies may have prevented infection. The development of an immune response that limits the effectiveness of repeat doses would be consistent with a number of studies in animals $(20,21,28)$. Moreover, even a modest immune response might have a significant impact on adenovirus infectivity because hexon neutralization of adenovirus infectivity has been reported to involve a single hit mechanism (29).

In contrast to the effects on $\Delta V_{\mathrm{t} \text { Low Cl}}$, basal $V_{\mathrm{t}}$ and $\Delta V_{\mathrm{t}}$ Amiloride were not altered. Previous in vitro studies using primary cultures of $\mathrm{CF}$ airway epithelia suggest that adenoviral vectors expressing CFTR can correct $\mathrm{Cl}^{-}$transport without correcting the increased $\mathrm{Na}^{+}$transport (7). Recently, Johnson et al. (8) showed that very high doses of an adenovirus vector expressing CFTR were required to reduce amiloride-sensitive current in cultured CF epithelia. Johnson et al. interpreted those results to suggest that expression of CFTR in a fraction of the cells of an epithelium may be sufficient to correct measures of $\mathrm{Cl}^{-}$transport (such as $\Delta V_{\mathrm{t} \text { Low Cl}}$ ) but not sufficient to normalize electrophysiologic properties that are influenced predominately by $\mathrm{Na}^{+}$transport (such as basal $V_{\mathrm{t}}$ and $\Delta V_{\mathrm{t}}$ Amiloride). All these data are consistent with the interpretation that, in nasal epithelia treated with Ad2/CFTR-2, only low levels of CFTR were synthesized or only a low percentage of cells expressed the protein. Although Ad2/CFTR-2 was able to correct the $\Delta V_{\mathrm{t} \text { Low Cl }}$ partially, we do not know the relationship between the correction of $\Delta V_{\mathrm{t} \text { Low Cl }}$ in an epithelium and the ability to prevent the progressive infections that are a major cause of morbidity and mortality.

Comparison of gene transfer with earlier studies. Two studies in humans with CF have shown that adenovirus vectors can express CFTR in airway epithelia as evaluated by reverse transcriptase-PCR and by immunohistochemical detection of protein $(13,14)$. We also showed that, after administering one dose of a related vector (Ad2/CFTR-1) to the nasal epithelium, CF-associated electrophysiologic abnormalities were partially corrected (13). We observed a decrease in basal $V_{\mathrm{t}}$ and a hyperpolarization of $V_{\mathrm{t}}$ during perfusion with terbutaline. In our present study, we did not find changes in basal $V_{\mathrm{t}}$ and we only observed changes consistent with generation of CFTR $\mathrm{Cl}^{-}$channels when we measured the more sensitive $\Delta V_{\mathrm{t}}$ Low $\mathrm{Cl}$. Because the changes we observed in the present study 

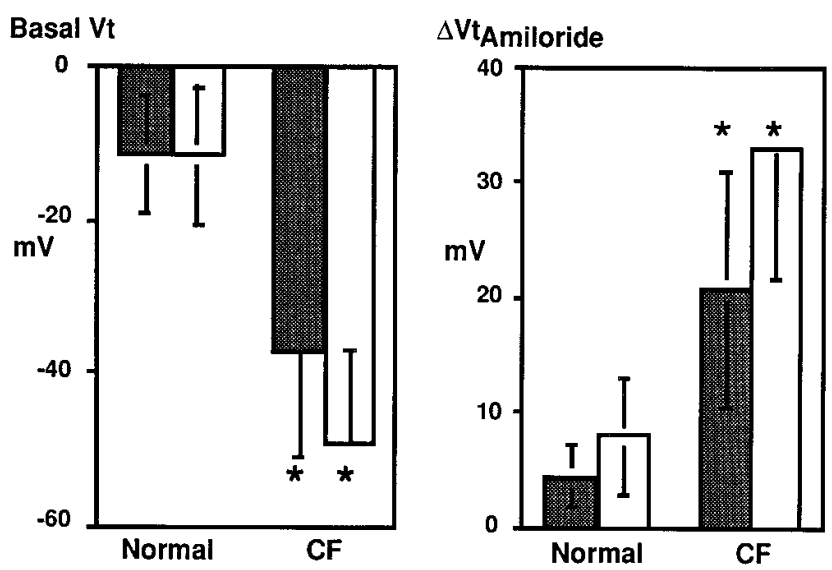

were less striking than in our first study (13), it is important to consider possible explanations. There are several factors to consider.

First, it is possible that there was an error in the first study. We think it unlikely that there were errors in measurement of $V_{\mathrm{t}}$ because basal $V_{\mathrm{t}}$, which is very easy to measure, changed over a prolonged time course in that study.

Second, although the vector and doses were different, it seems unlikely that that would explain the lesser effect detected here, because the administered doses were much higher in the present study.

Third, in the previous study, vector was administered on only one occasion, whereas in the present study each participant received multiple doses. It is possible that in our current study the initial doses of vector were not sufficient to effect correction but were able to stimulate an additional immune response that neutralized subsequently administered vector. However, the variability in electrophysiologic and immune response precludes a direct correlation between the two.

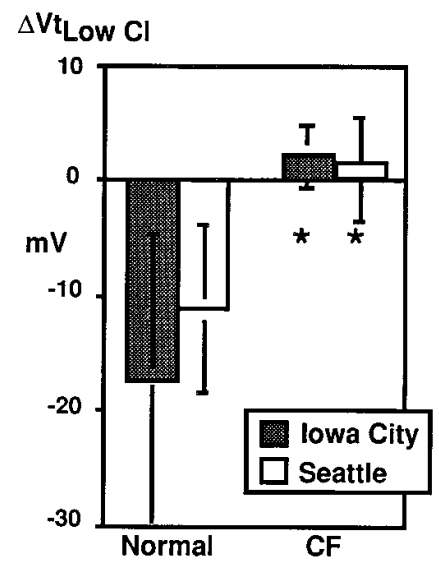

Figure 3. Electrophysiologic properties of normal and CF subjects. Data are mean \pm SD of subjects studied in Iowa City (shaded bars) and in Seattle (open bars). Data are basal $V_{\mathrm{t}}($ left $), \Delta V_{\mathrm{t}}$ Amiloride $(\mathrm{cen}$ ter), and $\Delta V_{\mathrm{t} \text { Low Cl }}($ right $)$ as described in Methods. $n=52$ assays in normal and 36 in $\mathrm{CF}$ in Iowa City, and 36 in normal and 70 in $\mathrm{CF}$ in Seattle. The comparison between normal and $\mathrm{CF}$ groups at each site was evaluated using an unpaired $t$ test. ${ }^{*} P<0.0001$.

Fourth, the site of administration was different in the two studies. In our first study, we applied vector to the medial aspect of the inferior turbinate because it was more accessible to the applicator. In the current study, we applied vector to the inferior aspect of the inferior turbinate and much of the nasal mucosa. The epithelium on the inferior aspect of the inferior turbinate is a pseudostratified, columnar, ciliated epithelium much like that in the large intrapulmonary airways. In contrast, cells on the medial aspect of the turbinate are more often a cuboidal or squamous nonciliated epithelium. Some studies suggest these types of cells have different susceptibility to adenovirus vectors (see below).

Fifth, in the first study we controlled the site and duration of exposure. By using an applicator in the first study, we constrained the vector to a small area $\left(\sim 0.5 \mathrm{~cm}^{2}\right)$ for a defined time (30 min). In our current study, the vector spread over the inferior turbinate. We estimate that the area of exposure was at least $10 \mathrm{~cm}^{2}$. When we applied methylene blue in $50 \mu \mathrm{l}$ using the present method, it spread rapidly to cover the entire nasal
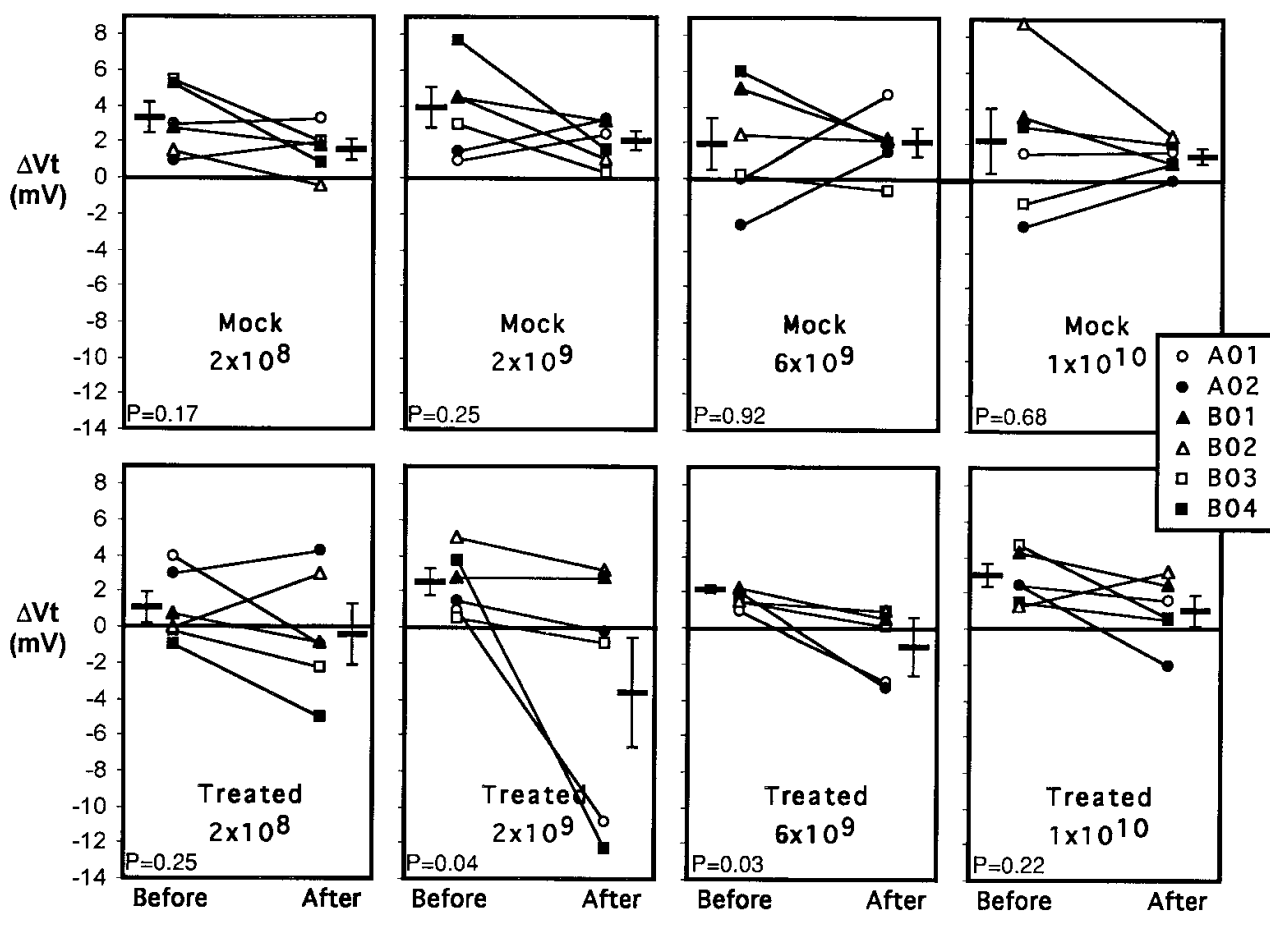

Figure 4. Change in voltage produced by perfusion of a solution containing a low $\mathrm{Cl}^{-}$concentration and terbutaline $\left(\Delta V_{\mathrm{t}}\right)$. Data are electrophysiologic measurements for individual subjects (each subject is indicated by a different symbol). Data points show average values before and for $1 \mathrm{wk}$ after vector administration. Pretreatment values are the average of two readings, one on day 0 for that administration and one on the most recent preceding day, and posttreatment values are the average of values for $1 \mathrm{wk}$ after vector administration (involving four readings on days $+1,+3,+5$, and $+7)$. Large bars and standard error bars on the sides of each panel show average changes in $\Delta V_{\mathrm{t}}$ for all six subjects. The administered dose is indicated at the bottom of each panel. Statistical significance was evaluated using the sign rank statistic; $P$ values are indicated in each panel. 
mucosa, whereas, when we applied methylene blue with an applicator, mucosal staining was limited to a discrete site. Moreover, we cannot determine the duration of contact of vector with the epithelium, which our unpublished studies indicate is an important determinant of the efficiency of gene transfer. In our first study, mucociliary clearance was probably inhibited by the use of topical cocaine and tetracaine (30).

Sixth, in our previous study we treated the nasal epithelium with vasoconstrictors and local anesthetics and applied the vector with a plexiglass spoon applicator for $30 \mathrm{~min}$. This treatment produced substantial local inflammation and injury that resolved over the subsequent $24 \mathrm{~h}$ (13). In the present study using a soft rubber catheter, no inflammation or irritation was observed. Other studies suggest that injured epithelium more readily takes up adenovirus vectors (12).

These results raise the question: How efficient are adenovirus vectors? Our first human study, in which moi's were in the 1-25 range, showed electrophysiologic evidence of correction. In the present study, the intermediate dose levels of $2-6 \times 10^{9}$, which showed partial correction, corresponded to an moi of 66-200 I.U./cell (assuming a target area of $10 \mathrm{~cm}^{2}$ and $3 \times 10^{6}$ cells $/ \mathrm{cm}^{2}$ ), whereas the highest dose corresponds to an moi of 330. Our earlier work using cultured human CF cells demonstrated robust correction of the $\mathrm{CF} \mathrm{Cl}^{-}$channel defect at a very modest moi (0.1-10) (7). More recently, we have observed that the efficiency of gene transfer decreases as the epithelium differentiates from a monolayer of cells with characteristics of basal cells to a more mature ciliated epithelium (Zabner, J., unpublished observation). Grubb et al. (12) have argued that mature human airway cells are indeed refractory to adenovirus-mediated gene transfer. If this is true in the lower pulmonary airways, where the number of target cells is $\sim 3 \times 10^{9}$, the doses of vector required to effect gene transfer even in the absence of an immune response could exceed levels that can be safely tolerated. For example, mouse experiments commonly use $1-5 \times 10^{9}$ I.U. in corresponding to an moi of 330-1,660, assuming $3 \times 10^{6}$ airway epithelial cells. The equivalent human dose would be $1-5 \times 10^{12}$ I.U.

Since submission of our work, there have been two other reports in which adenovirus encoding CFTR was delivered to the nasal epithelium of patients with CF. Hay et al. (31) administered one dose of adenovirus $\left(2 \times 10^{5}\right.$ to $2 \times 10^{8.5}$ plaqueforming units). After delivery they found partial correction of both the basal $V_{\mathrm{t}}$ and the $V_{\mathrm{t}}$ response to perfusion with a low $\mathrm{Cl}^{-}$concentration and isoproterenol, indicating at least partial complementation of the CF defect. Knowles et al. (32) delivered one dose $\left(2 \times 10^{7}-2 \times 10^{10}\right.$ plaque-forming units $)$ but were unable to show correction of either basal $V_{\mathrm{t}}$ or the response to low $\mathrm{Cl}^{-}$and isoproterenol. Moreover, at the highest dose there was mucosal inflammation in some subjects. It is difficult to compare directly the four different published studies in patients with CF (our present report plus references 11, 31,32 ) because there are many variables between the studies. However, we believe that the data suggest that improvement is needed for adenoviral vector-mediated gene transfer to human airway epithelia.

Future studies. Many important questions remain. For example, one obvious limitation of the present study is that it is the epithelium of the pulmonary airways, rather than the nasal mucosa, that is the ultimate target of gene transfer in $\mathrm{CF}$. Future studies will be required to test the safety of vector administered to the lung. In terms of the efficiency of gene transfer, we must learn more about the ability of adenovirus vectors to express CFTR in mature respiratory epithelium, and we must better understand the reasons for variability between subjects. Development of vectors that target ciliated airway epithelia may be of particular value in increasing the efficiency of gene transfer and as a result decreasing any associated adverse effects. Another important but unanswered question relates to the ability of gene transfer to correct not only the electrophysiologic abnormalities, but to check the progressive destruction of the airways in this lethal disease. These considerations suggest that substantial additional work in vitro, in animals, and in selected cases in humans are required for the development of successful gene transfer for patients with $\mathrm{CF}$.

\section{Acknowledgments}

We thank Aurita Puga, Terri McDonnell, Theresa Mayhew, Pary Weber, Lynne Keyes, Rosi Balfour, Lara Asmundson, Darshana Jani, Kathy Hehir, Judith St. George, Sharon McNamara, Guen McDonald, and Margaret Pepes for excellent assistance. We also thank members of the Genzyme Virus Production Group and the many colleagues involved in preclinical testing of Ad2/CFTR-2 and in the obtaining of regulatory approval to use it.

This work was supported by grants from the National Heart Lung and Blood Institute (Iowa), the Digestive Diseases and Kidney Institute (Washington), the Cystic Fibrosis Foundation, a General Clinical Research Center Program for the National Center for Research Resources, the Howard Hughes Medical Institute, and Genzyme Corporation.

\section{References}

1. Welsh, M.J., T.F. Boat, L.-C. Tsui, and A.L. Beaudet. 1995. Cystic fibrosis. In The Metabolic Basis of Inherited Disease. C.R. Scriver, A.L. Beaudet, W.S. Sly, and D. Valle, editors. McGraw-Hill, Inc., New York. 3799-3876.

2. Welsh, M.J., M.P. Anderson, D.P. Rich, H.A. Berger, G.M. Denning, L.S. Ostedgaard, D.N. Sheppard, S.H. Cheng, R.J. Gregory, and A.E. Smith. 1992. Cystic fibrosis transmembrane conductance regulator: a chloride channel with novel regulation. Neuron. 8:821-829.

3. Riordan, J.R. 1993. The cystic fibrosis transmembrane conductance regulator. Annu. Rev. Physiol. 55:609-630.

4. Collins, F.S. 1992. Cystic fibrosis: molecular biology and therapeutic implications. Science (Wash. DC). 256:774-779.

5. Rosenfeld, M.A., K. Yoshimura, B.C. Trapnell, K. Yoneyama, E.R Rosenthal, W. Dalemans, M. Fukayama, J. Bargon, L.E. Stier, L. Stratford-Perricaudet, M. Perricaudet, W.B. Guggino, A. Pavirani, J.P. Lecocq, and R.G. Crystal. 1992. In vivo transfer of the human cystic fibrosis transmembrane conductance regulator gene to the airway epithelium. Cell. 68:143-155.

6. Rich, D.P., L.A. Couture, L.M. Cardoza, V.M. Guiggio, D. Armentano, P.C. Espino, K. Hehir, M.J. Welsh, A.E. Smith, and R.J. Gregory. 1993. Development and analysis of recombinant adenoviruses for gene therapy of cystic fibrosis. Hum. Gene Ther. 4:461-476.

7. Zabner, J., L.A. Couture, A.E. Smith, and M.J. Welsh. 1994. Correction of cAMP-stimulated fluid secretion in cystic fibrosis airway epithelia: efficiency of adenovirus-mediated gene transfer in vitro. Hum. Gene Ther. 5:585-593.

8. Johnson, L.G., S.E. Boyles, J. Wilson, and R.C. Boucher. 1995. Normalization of raised sodium absorption and raised calcium-mediated chloride secretion by adenovirus-mediated expression of cystic fibrosis transmembrane conductance regulator in primary human cystic fibrosis airway epithelial cells. $J$. Clin. Invest. 95:1377-1382.

9. Rosenfeld, M.A., W. Siegfried, K. Yoshimura, K. Yoneyama, M. Fukayama, L.E. Stier, P.K. Paakko, P. Gilardi, L.D. Stratford-Perricaudet, M. Perricaudet, S. Jallat, A. Pavirani, J.-P. Lecocq, and R.G. Crystal. 1991. Adenovirus-mediated transfer of a recombinant $\alpha 1$-antitrypsin gene to the lung epithelium in vivo. Science (Wash. DC). 252:431-434.

10. Engelhardt, J.K.F., Y. Yang, L.D. Stratford-Perricaudet, E. D. Allen, K. Kozarsky, M. Perricaudet, J.R. Yankaskas, and J.M. Wilson. 1993. Direct gene transfer of human CFTR into human bronchial epithelia of xenografts with E1deleted adenoviruses. Nat. Genet. 4:27-34.

11. Zabner, J., D.M. Petersen, A.P. Puga, S.M. Graham, L.A. Couture, L.D. Keyes, M.J. Lukason, J.A. St. George, R.J. Gregory, A.E. Smith, and M.J. Walsh. 1994. Safety and efficacy of repetitive adenovirus-mediated transfer of CFTR cDNA to airway epithelia of primates and cotton rats. Nat. Genet. 6:75-83. 
12. Grubb, B.R., R.J. Pickles, H. Ye, J.R. Yankaskas, R.N. Vick, J.F. Engelhardt, J.M. Wilson, L.G. Johnson, and R.C. Boucher. 1994. Inefficient gene transfer by adenovirus vector to cystic fibrosis airway epithelia of mice and humans. Nature (Lond.). 371:802-806.

13. Zabner, J., L.A. Couture, R.J. Gregory, S.M. Graham, A.E. Smith, and M.J. Welsh. 1993. Adenovirus-mediated gene transfer transiently corrects the chloride transport defect in nasal epithelia of patients with cystic fibrosis. Cell. 75:207-216.

14. Crystal, R.G., N.G. McElvaney, M.A. Rosenfeld, C.S. Chu, A. Mastrangeli, J.G. Hay, S.L. Brody, H.A. Jaffe, N.T. Eissa, and C. Danel. 1994. Administration of an adenovirus containing the human CFTR cDNA to the respiratory tract of individuals with cystic fibrosis. Nat. Genet. 8:42-51.

15. McElvaney, N.G., and R.G. Crystal. 1995. IL-6 release and airway administration of human CFTR cDNA adenovirus vector. Nat. Med. 1:182-184

16. Simon, R.H., J.F. Engelhardt, Y. Yang, M. Zepeda, S. Weber-Pendleton, M. Grossman, and J.M. Wilson. 1993. Adenovirus-mediated transfer of the CFTR gene to lung of nonhuman primates: toxicity study. Hum. Gene Ther. 4: 771-780.

17. Brody, S.L., M. Metzger, C. Danel, M.A. Rosenfeld, and R.G. Crystal. 1994. Acute responses of non-human primates to airway delivery of an adenovirus vector containing the human cystic fibrosis transmembrane conductance regulator cDNA. Hum. Gene Ther. 5:821-836.

18. Bout, A., J.-L. Imler, and H. Schultz. 1994. In vivo adenovirus-mediated transfer of human CFTR cDNA to Rhesus monkey airway epithelium: efficacy, toxicity and safety. Gene Ther. 1:385-394.

19. Yei, S., N. Mittereder, S. Wert, J.A. Whitsett, R.W. Wilmott, and B.C. Trapnell. 1994. In vivo evaluation of the safety of adenovirus-mediated transfer of the human cystic fibrosis transmembrane conductance regulator cDNA to the lung. Hum. Gene Ther. 5:731-744.

20. Yang, Y., F.A. Nunes, K. Berencsi, E.E. Furth, E. Gonczol, and J.M. Wilson. 1994. Cellular immunity to viral antigens limits E1-deleted adenoviruses for gene therapy. Proc. Natl. Acad. Sci. USA. 91:4407-4411.

21. Kaplan, J.M., J.A. St. George, S.E. Pennington, L.D. Keyes, P. Johnson, S.C. Wadsworth, and A.E. Smith. 1995. Humoral and cellular immune responses of non-human primates to long term repeated lung exposure to Ad2/ CFTR-2. Gene Ther. In press.
22. Yang, Y., F.A. Nunes, K. Berencsi, E. Gonczol, J.F. Engelhardt, and J.M. Wilson. 1994. Inactivation of E2a in recombinant adenoviruses improves the prospect for gene therapy in cystic fibrosis. Nat. Genet. 7:362-369.

23. Knowles, M., J. Gatzy, and R. Boucher. 1981. Increased bioelectric potential difference across respiratory epithelia in cystic fibrosis. N. Engl. J. Med. 305:1489-1495.

24. Alton, E.W., J.G. Hay, C. Munro, and D.M. Geddes. 1987. Measurement of nasal potential difference in adult cystic fibrosis, Young's syndrome, and bronchiectasis. Thorax. 42:815-817.

25. Welsh, M.J., J. Zabner, S.M. Graham, A.E. Smith, R. Moscicki, and S.C Wadsworth. 1995. Adenovirus-mediated gene transfer for cystic fibrosis. Part A. Safety of dose and repeat administration in the nasal epithelium. Part B. Clinical efficacy in the maxillary sinus. Hum. Gene Ther. 6:205-218.

26. Ramsey, B., and M.A. Richardson. 1992. Impact of sinusitis in cystic fibrosis. J. Allergy Clin. Immunol. 90:547-552.

27. Armentano, D., C.C. Sookdev, K.M. Hehir, R.J. Gregory, J.A. St.George, G.A. Prince, S.C. Wadsworth, and A.E. Smith. 1995. Characterisation of an adenovirus gene transfer vector containing an E4 deletion. Hum. Gene Ther. In press.

28. Yei, S., N. Mittereder, K. Tang, C. O'Sullivan, and B.C. Trapnell. 1994. Adenovirus-mediated gene transfer for cystic fibrosis: quantitative evaluation of repeated in vivo vector administration to the lung. Gene Ther. 1:192-200.

29. Wohlfart, C. 1988. Neutralization of adenoviruses: kinetics, stoichiometry, and mechanisms. J. Virol. 62:2321-2328.

30. Ingels, K.J., M.R. Nijziel, K. Graamans, and E.H. Huizing. 1994. Influence of cocaine and lidocaine on human nasal cilia. Beat frequency and harmony in vitro. Arch. Otolaryngol. Head \& Neck Surg. 120:197-201.

31. Hay, J.G., N.G. McElvaney, J. Herena, and R.G. Crystal. 1995. Modification of nasal epithelial potential differences of individuals with cystic fibrosis consequent to local administration of a normal CFTR cDNA adenovirus gene transfer vector. Hum. Gene Ther. 6:1487-1496.

32. Knowles, M.R., K.W. Hohneker, Z. Zhou, J.C. Olsen, T.L. Noah, P.-C. Hu, M.W. Leigh, J.F. Engelhardt, L.J. Edwards, K.R. Jones, et al. 1995. A controlled study of adenoviral-vector-mediated gene transfer in the nasal epithelium of patients with cystic fibrosis. N. Engl. J. Med. 333:823-831. 